\section{Zika Virus: Facts, Prevention Strategies, and an Informed Tourist Perspective}

Before we left Qatar for Brazil on holidays, only a few news reports had reached us about a virus spreading throughout the Americas. However, after spending 2 weeks at the centre of the outbreak, we gained a unique perspective on the public health crisis affecting Central and South America. This article summarizes, up to May 2016, knowledge of the Zika virus, provides evidence-based recommendations regarding prevention of Zika infection, and provides a perspective on issues related to travel medicine for pharmacists outside of Zika-active zones.

As of February 2016, the World Health Organization had declared the spread of Zika virus a public health emergency of international concern, a verdict that should prompt international action and research priorities. ${ }^{1}$ Zika virus is a flavivirus, with mosquitoes serving as the vectors of transmission, which causes effects similar to those of other well-known viruses, such as yellow fever virus, dengue virus, and West Nile virus. ${ }^{2}$ Aedes mosquitos are deemed to be the primary transmitting vectors, but other vectors cannot be ruled out. ${ }^{3}$ Sexual transmission and congenital cases have also been documented. Alarmingly, this virus is spreading quickly among several countries across multiple continents, including Central America, the Caribbean, Mexico, and South America. The clinical presentation is largely asymptomatic, yet some patients experience mild, nonspecific flu-like symptoms such as fever, chills, rash, conjunctivitis, and joint pain. Treatment consists only of supportive measures. ${ }^{4}$

Available data give a high suspicion for the association of Zika virus with development of neurological disorders, including congenital microcephaly and Guillain-Barré syndrome. ${ }^{2}$ As such, pregnant women are considered a high-risk population. Few studies to date suggest causality; however, observational data associate small fetal head size and brain calcifications with Zika infection. Between October 2015 and January 2016, in Brazil alone, about 4000 babies were born with microcephaly, which represents a 20 -fold increase from the number of cases occurring between 2010 and 2014. ${ }^{5}$

Effective prevention strategies are key to control of virus transmission and spread. These strategies can be divided into 2 broad categories: those affecting mosquito population control and those targeting individuals. Focusing efforts to control mosquito populations in endemic regions should be a priority; however, vector-control strategies employed to control dengue and chikungunya viruses within affected regions have failed in the past. ${ }^{6}$ Efforts must be coordinated to effectively reduce the burden of carrier mosquitos and subsequently the rates of transmission to humans. Unfortunately, no vaccine to prevent Zika transmission is available, nor will any be available in the near future.

Tourists and those living within endemic regions should take precautions against mosquito bites. Currently, the US Centers for Disease Control and Prevention (CDC) recommends use of mosquito repellent containing $N, N$-diethyl-3-meta-toluamide (DEET), picaridin, oil of lemon eucalyptus, or IR3535. One study showed that products with DEET were most effective for the longest duration. ${ }^{8}$ Recommendations also include wearing clothing that covers the arms and legs and/or using permethrin-treated clothing. ${ }^{7}$ Additional measures may include using insecticide-treated mosquito nets when sleeping and minimizing time in areas of high mosquito volume. Postponement of travel plans to Zika-active areas can also be considered.

The advent of sexual transmission prompted the CDC to release interim guidelines for prevention in early 2016. ${ }^{9}$ These guidelines are very important from a public health perspective, as the virus may persist longer in semen than in blood..$^{10}$ The CDC

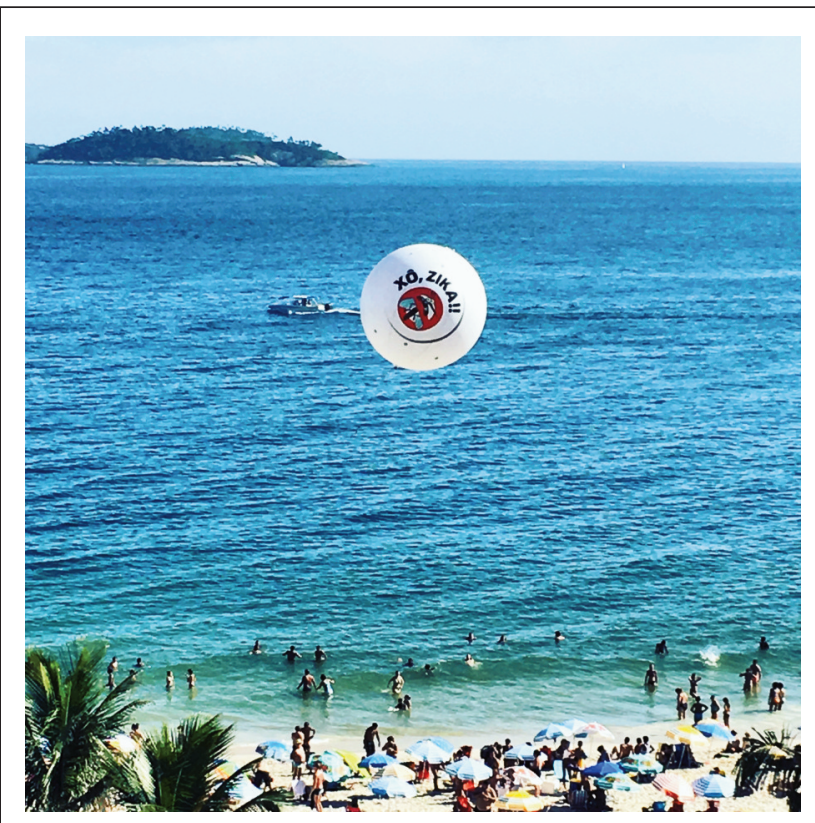

Figure 1. Zika awareness campaign, using a beach balloon, on Ipanema Beach, Rio de Janeiro, Brazil. This photograph provides an example of a health promotion program targeted to residents and tourists in Brazil. Increased efforts are needed in Zika-active zones to provide people with information about effective mosquito-prevention strategies. Photo courtesy of Kyle Wilby. 
advises men who reside in or have travelled to a Zika-active area and whose partners are pregnant to abstain from sexual activity or to use condoms consistently and correctly during oral, vaginal, and anal intercourse. For men who reside in or have travelled to a Zika-active area and whose partners are not pregnant, it is advised that they consider abstaining from sexual activity or using condoms consistently and correctly during sex. ${ }^{9}$

As "informed tourists" in Brazil, we observed attempts at raising awareness through media and in public spaces (Figure 1). However, information about the potential seriousness of the infection and appropriate prevention strategies was not readily available. Specifically, mosquito repellent was not easy to find and, once located (inside a community pharmacy), products were limited in terms of both number and selection. Therefore, we urge health professionals encountering patients who are planning to visit Zikaactive areas to educate the travellers about the disease and to encourage them to purchase repellents and other prevention products and clothing before travel. Pharmacists must also be diligent in questioning returning patients about potential exposure to the Zika virus and must learn to recognize signs and symptoms consistent with infection.

Despite best efforts to prevent Zika virus disease at an individual patient level, a coordinated global strategy for virus control is urgently needed. Such a strategy is especially important as Brazil prepares to host the 2016 Summer Olympic Games and as travel to active zones increases in the coming months.

\section{References}

1. WHO Director-General summarizes the outcome of the Emergency Committee regarding clusters of microcephaly and Guillain-Barré syndrome [media statement]. Geneva (Switzerland): World Health Organization; 2016 Feb 1 [cited 2016 Feb 13]. Available from: www.who.int/mediacentre/news/ statements/2016/emergency-committee-zika-microcephaly/en/

2. Zika virus [fact sheet]. Geneva (Switzerland): World Health Organization; 2016 Apr 15 [cited 2016 May 13]. Available from: www.who.int/ mediacentre/factsheets/zika/en/

3. Ayres CF. Identification of Zika virus vectors and implications for control. Lancet Infect Dis. 2016;16(3):278-9.

4. MacFadden DR, Bogoch II. Zika virus infection. CMAJ. 2016;188(5):367.

5. Gulland A. WHO urges countries in dengue belt to look out for Zika. BMJ. 2016;352:i595.

6. Rubin EJ, Greene MF, Baden LR. Zika virus and microcephaly. $N$ Engl J Med. 2016;374(10):984-5.

7. Zika virus in South America. Atlanta (GA): Centers for Disease Control and Prevention (US); 2016 [cited 2016 May 13]. Available from: wwwnc. cdc.gov/travel/page/zika-travel-information

8. Fradin MS, Day JF. Comparative efficacy of insect repellents against mosquito bites. N Engl J Med. 2002;347(1):13-8.

9. Oster AM, Russell K, Stryker JE, Friedman A, Kachur RE, Peterson EE, et al. Update: interim guidance for prevention of sexual transmission of Zika virus - United States, 2016. MMWR Morb Mortal Wkly Rep. 2016; 65(12):323-5.

10. Musso D, Roche C, Robin E, Nhan T, Teissier A, Cao-Lormeau VM. Potential sexual transmission of Zika virus. Emerg Infect Dis. 2015;21(2): 359-61. Erratum in: Emerg Infect Dis. 2015;21(3):552.

Kyle J Wilby, BSP, ACPR, PharmD

Ziad G Nasr, BSC(Pharm), PharmD, BCPS

College of Pharmacy

Qatar University

Doha, Qatar

Competing interests: None declared. 